Original Research

\title{
Test-Retest Reliability of the Isometric Soleus Strength Test in Elite Male Academy Footballers
}

David Rhodes, PhD, MSc, MSST ${ }^{1}$ (1) ${ }^{\text {, }}$, Josh Jeffery ${ }^{2}$, Daniel Brook-Sutton, BSc, MSST ${ }^{3}$, Jill Alexander, MSc, MSST ${ }^{1}$

${ }^{1}$ Football Performance Hub, Institute of Coaching and Performance (ICaP), School of Sport and Health Sciences, University of Central Lancashire, 2 Medical and Performance, Everton Football Club, ${ }^{3}$ Sport, Nutrition and Clinical Sciences, School of Sport and Health Sciences, University of Central Lancashire

Keywords: soleus, isometric, reliability, muscle strength, soccer, football

https://doi.org/10.26603/001c.31047

\section{International Journal of Sports Physical Therapy}

Vol. 17, Issue 2, 2022

\section{BACKGROUND}

Currently there is no reliability data available for the isometric soleus strength test (ISST), commonly used as a monitoring tool in elite football settings. Isometric strength testing for other muscle groups, most notably the hamstrings, is utilized to identify injury risk and readiness to train/play. To profile athletes efficiently, performance practitioners require optimal measures that are reliable. The aim of this study was to investigate the test-retest reliability of the isometric strength test of the soleus and propose a standardized protocol for its use within an elite male football population.

\section{STUDY DESIGN}

Test-retest reliability single cohort study.

\section{METHODS}

Thirty elite male footballers (age $=22.8 \pm 5.0$ years, height $=180.0 \pm 0.08 \mathrm{~cm}$, weight $=$ $70.57 \pm 4.0 \mathrm{~kg}$ ) performed the ISST, through three maximum 3-second hold efforts with one minute rest between repetitions and 48 hours between tests, in each test. The test was performed mid-competitive season. All data bilaterally were checked for normality using the Shapiro-Wilk test before a Pearson's Correlations and Bland-Altman's analyses were performed.

\section{RESULTS}

Test-retest reliability demonstrated high reliability for ISST bilaterally (Right: $\mathrm{r}=0.89$; Left: $r=0.79, p<0.05)$. The standard error of measurement (SEM) (\%) was $9.09-12.47 \%$ and minimal detectable change (MDC) was 25.19 - $34.56(\mathrm{~N})$ for Peak Force $(\mathrm{PF})$ measures of the ISST. Bilateral levels of agreement were found to be $+/-2$ standard deviations (SD) of the interval of agreement bilaterally for ISST (Levels of agreement (LOA): Right: Upper 352.49 - Lower -494.76; Left: Upper 523.82 - Lower -591.30. Bilaterally no significant difference was detected between values (Right: $p=0.09$, CI: $-153.21-10.95$; Left: $p=0.52$, CI: -139.81-72.33).

\section{CONCLUSION}

The results of this study demonstrate high reliability for the ISST. The ISST displays a high test-retest reliability for assessing PF characteristics of the soleus in elite male

\footnotetext{
a Corresponding Author:

David Rhodes

Football Performance Hub, Institute of Coaching and Performance

University of Central Lancashire

Fylde Road

Preston

PR1 2HE

drhodes2@uclan.ac.uk

01772895491
} 
academy footballers. This test may be beneficial for performance practitioners for profiling soleus function of athletes.

\section{INTRODUCTION}

An important issue in elite football settings is quantifying muscle function for performance. Identifying muscular dysfunction/weakness through appropriate testing related to injury aetiology provides practitioners with valuable information to intervene with appropriate programs to address these issues. Such programs may mitigate the negative impact injuries can have on team performance through time loss and financial cost. ${ }^{1}$ The physical demands of football (soccer) are known to have increased ${ }^{2}$ and risk of injury is high. ${ }^{3,4}$ Consequently, performance practitioners in elite settings aim to implement testing protocols to identify an athlete's readiness to train/play, maximising performance and minimising potential injury risk factors. Muscle strength has been a key etiological factor associated with injury risk. ${ }^{5,6}$ Muscle injuries are a predominant feature in many football-related investigations. ${ }^{7}$ The use of isometric testing to identify reductions in muscle strength is common practice, ${ }^{8}$ and integral for the decision-making process that takes place on a daily basis in an elite football setting to maximize performance and increase player availability.

Literature has focussed heavily on quantifying hamstring and quadricep muscle function and the reliability of such measures are well reported.5,6 This has led to widespread use of these measures in practical settings, particularly in elite football, where muscle testing equipment is widely available. This focus has been predominantly driven by injury occurrence, identifying risk in athletes, and increasing performance. Occurrence of posterior lower leg injuries is prevalent in elite football and team sports. ${ }^{4,9}$ It is important to note the key contribution of the posterior lower leg, particularly the soleus, on running performance, ${ }^{10-12}$ highlighting the need within elite sport to identify reliable methods to quantify soleus function. Reliable quantification methods are essential for effective monitoring or athlete profiling and could provide practitioners with data that can influence decision making in relation to readiness to train/play, training prescription and rehabilitation processes. ${ }^{13}$

Isometric muscle strength testing is commonly utilized to determine musculoskeletal status, markers for return to play and address strength deficits affecting performance. ${ }^{14}$ The use of isometric muscle strength testing is commonly utilized within elite sport, due to it being less provocative than eccentric testing. ${ }^{15,16}$ This allows testing to be completed more frequently and during heavy fixture congested periods, identifying any deficits that may be associated with reductions in performance or potential injury risk factors. Isometric contractions are a highly reliable and efficient way of measuring and monitoring changes in the generation of force. ${ }^{17}$ The isometric soleus strength test (ISST) is utilized to determine the strength of plantar flexors, notably the soleus muscle. No standardization of testing or reliability data is available to the authors knowledge for the ISST. A standardized and reliable measure that can be utilized to test for isometric soleus strength may provide medical and performance practitioners with the utility to op- timally monitor and profile athletes. Therefore, the aim of this study was to investigate the test-retest reliability of the isometric strength test of the soleus and propose a standardized protocol for its use within an elite male football population.

\section{MATERIALS AND METHODS}

\section{PARTICIPANTS}

An a priori power calculation using G-power indicated that a total of 30 participants would be required to detect a high correlation with an alpha of $5 \%$ and power of $80 \%$. Thirty male elite academy footballers (age $=22.76 \pm 5.0$ years, height $=180.0 \pm 0.8 \mathrm{~cm}$, weight $=70.57 \pm 4.0 \mathrm{~kg}$ ) participated in this study during the 2019-2020 season. Participants were advised of the advantages and risks of the study and the testing protocol was clearly defined verbally before participants provided written and verbal consent to participate, with the option to withdraw from testing at any point. Participants had a minimum of four years' experience in resistance training and strength-based testing protocols and met the inclusion criteria of healthy with no current injury and were of male gender. All players eligible for the study were in full training, free from injury, and available for competitive selection. The host football club permitted the dissemination of anonymous data for publication of the study findings and the study commenced in accordance with the 2013 Declaration of Helsinki and was approved by the University of Central Lancashire ethical committee (STEMH).

This study evaluated the test-retest reliability of the ISST within an elite male academy football population using a correlation design. Data collection was performed in a temperature-controlled physiology laboratory on site at the host football club training ground by the same two researchers throughout. Testing occurred at the same time of day for the re-test data $72 \mathrm{hrs}$ apart to account for potential diurnal or circadian rhythm that could have affected performance. ${ }^{18}$ Players refrained from strenuous exercise between these two testing periods and completed their normal daily routine.

\section{STUDY DESIGN}

Participants were familiar with the test protocol, as it has been utilized within the previous season consistently throughout the clubs' regular screening, testing and readiness to train/play protocols. Testing for the present study took place within pre-season. Test procedures for the ISST were appropriately standardized following previous recommendations in the literature. ${ }^{19}$ Before the commencement of ISST bar height was determined for each individual participant, based on seating position and maintenance of hip, knee and ankle joints at 90-degrees, in order to achieve the correct body position for each test. ${ }^{19}$ Seating position, rack bar, crocodile pin and bar position were recorded for protocol standardization ahead of scheduled testing. Procedures were identical between both testing sessions. Although there is no standardized warm up for the ISST, it 
Table 1. Isometric Soleus Strength Test (ISST) strength measures $(n=30)$ and reliability statistics

\begin{tabular}{|c|c|c|c|c|c|c|c|c|}
\hline TEST & LIMB & Mean Test \pm SD & $\begin{array}{c}\text { Mean Retest } \pm \\
\text { SD }\end{array}$ & $(95 \% \mathrm{Cl})$ & $\begin{array}{c}r \\
\text { value }\end{array}$ & $\begin{array}{l}\text { SEM } \\
\text { (N) }\end{array}$ & SEM\% & $\begin{array}{c}\text { MDC } \\
\text { (N) }\end{array}$ \\
\hline ISST & Right & $\begin{array}{c}1775.1 \pm 486.7 \\
(\mathrm{~N})\end{array}$ & $\begin{array}{c}1846.27 \pm 391.6 \\
(N)\end{array}$ & $\begin{array}{l}(0.78, \\
0.96)\end{array}$ & 0.89 & 161.41 & 9.09 & 25.19 \\
\hline ISST & Left & $\begin{array}{c}1733.9 \pm 471.9 \\
(\mathrm{~N})\end{array}$ & $\begin{array}{c}1767.6 \pm 327.0 \\
(\mathrm{~N})\end{array}$ & $\begin{array}{l}(0.70 \\
0.93)\end{array}$ & 0.79 & 216.24 & 12.47 & 34.56 \\
\hline
\end{tabular}

ISST, Isometric Soleus Strength Test, SD, standard deviation, Pearson's correlation r value, CI, confidence interval, $\mathrm{N}=$ Newtons, SEM, standard error of measurement, MDC, minimal detectable change.

is apparent from other isometric tests that a derivative of the movement performed should be incorporated. $15,16,19,20$ Participants therefore completed a standardized warm-up beginning with a 10-minute of supervised stationary cycling $1.5 \mathrm{~W} \mathrm{~kg}-1$, cadence of $60 \mathrm{rpm}$ on a cycle ergometer (Wattbike Ltd, Nottingham, UK), followed by five minutes of dynamic stretching, before advancing to two warm-up sets of IMTP soleus lifts at $50 \%$ and $75 \%$.

The BTS-6000 force decks platform (VALD Performance, Newstead, Queensland Australia) were calibrated by the manufacturer to evenly distribute bodyweight across the two platforms. The participant was seated in a position of 90-degrees hip and knee flexion, feet hip-width apart and of equal distance from the center of the platform. While the use of a "self-selected" body position is likely to be advantageous for testing performance, it is not recommended without ensuring that the hip, knee, and ankle joint angles are at 90 degrees, due to the potential influence of varied body positions on force generation. ${ }^{19,21}$ An Airex (Airex AG Sins, Switzerland) cushion was placed on top of the participants' thighs, with the bar placed on top. The individual was then asked to position the metal bar in line with their pre-recorded position, within the crocodile pins on the Sorinex XL racking system (Sorinex, Lexington, SC, USA), with the bar placed directly over the lateral malleolus. Participants were encouraged to maintain a vertical posture throughout the movement, with hands away from the bar due to interference previously recognized. Before each test, this position was ascertained, with the knee joint angle verified using a goniometer. The width of the participants' foot position was measured using a standard measuring tape to ensure consistency between tests. After performing two warm-up efforts for the ISST at 50 and $75 \%$, the participant performed three maximum efforts (three second hold with a one-minute rest between reps). Participants were advised to maintain a neutral foot position and minimal pre-tension on the bar until verbal instruction was given. Before each rep the athlete was guided by a countdown (“ $3,2,1$ ”) and instructed to push for three seconds up and against the bar as hard and as fast as possible. ${ }^{18}$

\section{DATA ANALYSIS}

Initial data analysis was performed using Forcedecks software (VALD Performance, Newstead, Queensland Australia) and transferred to a spreadsheet program (Microsoft Excel,
Microsoft Corp., Redmond, WA, USA). Data was recorded for each of the three maximum efforts of three seconds over the two sessions utilized to ascertain reliability of the test. An average was taken for each participant and relative reliability was calculated to identify the relationship for each limb. A Pearson's correlation measured the relationship between the two testing sessions. The following criteria quantified magnitude of the correlation $<0.1$, trivial; $>0.1$ to 0.3 , small; $>0.3$ to 0.5 , moderate; $>0.5$ to 0.7 , large; $>0.7$ to 0.9 , very large; and $>0$ to 1.0 , almost perfect, with statistical significance set at $p \leqslant 0.05$. Reliability in units of measurement was calculated for the interpretation of group mean scores and the individual scores of Peak Force (PF) (N) including Standard error of measurement (SEM) and minimal detectable change (MDC). The formulas used for both SEM and MDC followed previous calculations described by Ransom et al. ${ }^{14}$ To analyze for levels of agreement a BlandAltman method was completed. ${ }^{22}$ Prior to completing statistical analyses the distribution of data was assessed for normality using the Shapiro-Wilk Test and found to be suitable for parametric statistical testing. All statistical analysis was completed utilising SPSS software version 26.0 (SPSS, Chicago, IL, USA)

\section{RESULTS}

A significant correlation was demonstrated between tests $(p<0.001)$. A very large correlation demonstrated for the ISST (Right: $r=0.89$; Left: $r=0.79$ ) (Table 1). Figure 1 highlights the linear relationship in the reliability data, bilaterally.

Figure $\underline{2}$ and $\underline{3}$ display the mean differences between the test-retest data for the ISST with the upper and lower 95\% confidence intervals displayed for the measures taken.

Bilateral levels of agreement were found to be +/- 2 standard deviations (SD) of the interval of agreement bilaterally for ISST (Levels of agreement (LOA): Right: Upper 352.49 Lower -494.76; Left: Upper 523.82 - Lower -591.30. No significant difference between the mean scores for the right $(p=0.09, \quad C I:-153.21-10.95)$ or left $(p=0.52, \quad C I$ : -139.81-72.33) test-retest mean scores were found, indicating that high levels of agreement were identified between the two tests bilaterally. 


\section{DISCUSSION}

The aim of the current study was to evaluate the reliability of the ISST in an elite academy football population. It was hypothesised that reliability would be high for the ISST in this population. The primary findings from this study demonstrated high reliability in male academy footballers for the ISST. High levels of agreement were demonstrated between the two ISST tests bilaterally with $95 \%$ of differences demonstrated to be less than two standard deviations away from the mean. ${ }^{22}$ Indicating that the ISST can be utilized in an elite sporting environment to reliably quantify isometric strength of the soleus. The SEM (\%) and MDC values indicated absolute reliability across measures suggesting changes in strength in an individual athlete can be determined from this test (Table 1).

Isometric strength testing is commonly used for identifying modifiable injury risk factors or assessing outcomes of performance enhancement programs. Confidence within the test in terms of repeatability is important for sports medicine and performance practitioners to consider. The ability to evaluate an athlete's lower limb capacity to generate force is an integral part of strength profiling and evaluating the efficacy of training interventions. ${ }^{23}$ The strength of plantar flexors, notably the soleus muscle may be determined through the ISST. The test position in $80-90^{\circ}$ knee flexion has been shown to inhibit the force generated by gastrocnemius, therefore primarily evaluating the strength of the soleus. ${ }^{19}$ Currently no standardised testing protocol exists for ISST. Recently Ransom et al. ${ }^{14}$ indicated the importance of repeatability in terms of detecting true changes in response to injury or load over time in order to enhance athlete profiling. Consequently, it is important to report both test-retest and absolute reliability. This provided assessment of variability between repeated measures as well level of agreement in PF data. ${ }^{14}$ Current results provide confidence in the measure of isometric soleus muscle strength through the ISST by highlighting normal variance and levels of agreement between testing sessions. ${ }^{22}$ Evidently, isometric soleus PF measures in the current study were reproducible both between sessions. Results from the current study on isometric soleus strength testing, support similar findings by De Witt ${ }^{24}$ and Haff et al. ${ }^{25}$ The current study offers further evidence that ISST PF is a useful metric for reliably quantifying maximum strength from isometric strength test protocols.

Medical and performance practitioners working within an elite performance setting may consider using the ISST to evaluate athletes' optimum and PF capabilities due to the high reliability of the test identified in the current study. Practitioners may be reluctant to conduct maximal eccentric strength testing due to the potential risk of injury. Thus, prompting the need to consider the implementation of the ISST as an alternative measure to determine maximum strength and/or PF. The ISST being isometric in nature, decreases the risk of fatigue and subsequent risk of injury, thus providing a measure that can be utilized in fixture congested periods. ${ }^{24}$ Earlier researchers have advocated for in-depth analysis of players which should include isometric muscle strength (albeit in the hamstrings as an example) ${ }^{26}$ which may influence optimal training prescription. ${ }^{27}$ From

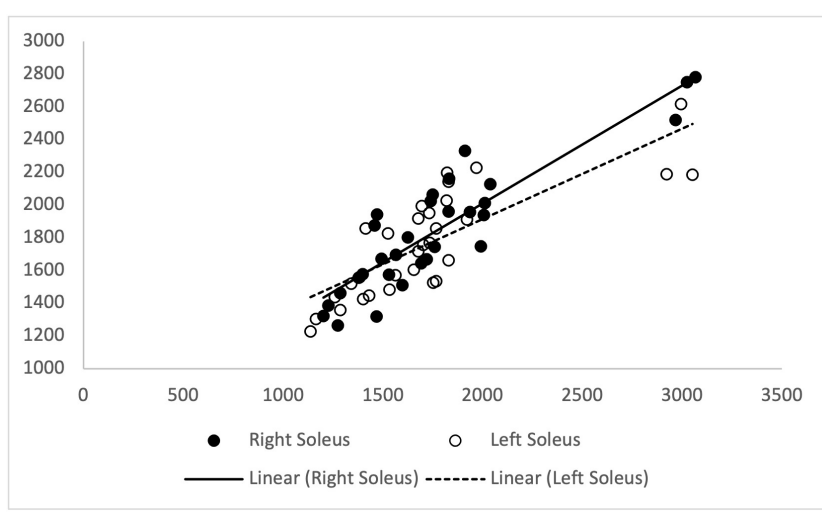

Figure 1. Test-Retest Relationships for the Isometric Soleus Strength Test (ISST)

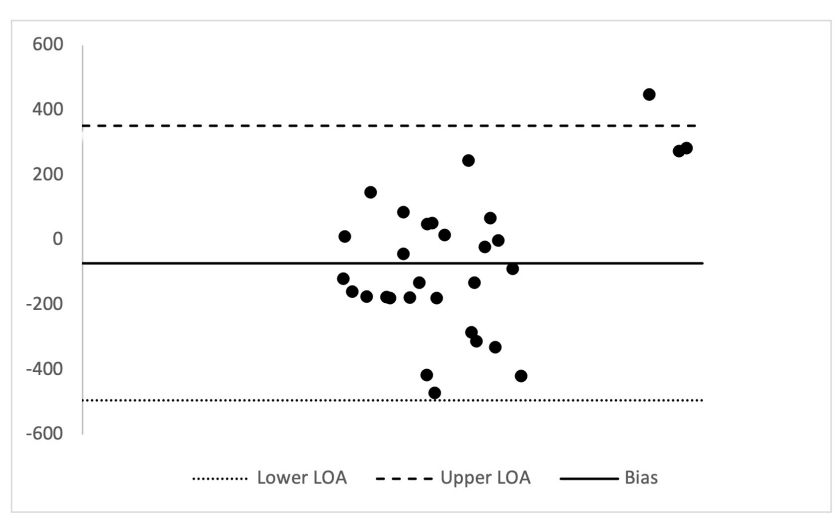

Figure 2. Bland-Altman Analysis for the Right ISST Displaying the Mean Difference between the testretest and the $95 \%$ Confidence Limits for the Measures (Upper and Lower Levels of Agreement [LOA])

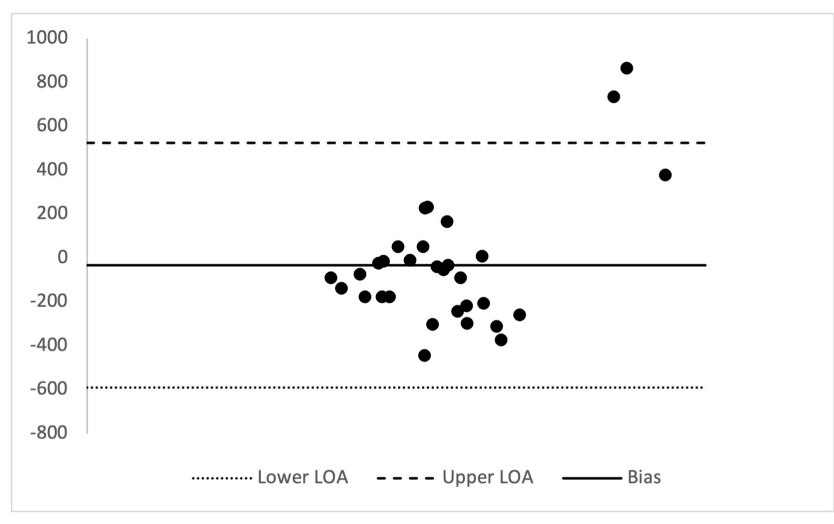

Figure 3. Bland-Altman Analysis for the Left ISST Displaying the Mean Difference between the testretest and the $\mathbf{9 5 \%}$ Confidence Limits for the Measures (Upper and Lower Levels of Agreement [LOA])

an applied perspective ISST quantifies vertical forces which are essential mechanical components for specific athletic 
functions such as acceleration, sprinting, distance jumping and directional changes. ${ }^{28}$ Reliable screening techniques such as the ISST may support the identification of at-risk or strength deficient players and consequently the adaptation of preventative interventions or programs targeting individuals can be derived from such information. This initial study only considered PF as a metric, due to its strong association to functional activities and movement patterns. ${ }^{19}$ Any future work in the area should consider other metrics, such as rate of force development (RFD).

SEM (\%) and MDC data provided measurement error for absolute reliability (Table 1). Analysis of PF data before and after interventions may be assisted by the MDC data from the current study findings. For the ISST, SEM demonstrated 161.41-216.24N and a small relative index of $9.09-$ $12.47 \%$, with any individual PF changes above $25.19-34.56 \mathrm{~N}$ being expected to be a 'real' change. Further investigation in other populations and ages within elite or normative populations may be beneficial to determine agreement. Although this type of data may provide sports medicine and performance practitioners with guidance on 'real' changes in strength that may occur, as presented in recent similar studies. ${ }^{14}$

Due to the high reliability demonstrated within the present study the ISST for soleus this metric may be a useful objective marker for quantifying posterior lower limb function. Furthermore, the equipment allows for assessment between dominant and non-dominant legs through metrics derived from the Forceplate software (VALD Performance, Newstead, Queensland Australia). Future studies may consider further investigation of dominant or non-dominant limb through utilization of the ISST. For example, recovery of lower limb muscle strength in the dominant leg is re- ported to be compromised for up to 72 hours after competitive fixture. ${ }^{6,29}$ While a specific definition of muscle imbalance has yet to be agreed upon, debate continues as to the contribution of imbalance as a risk factor for professional football injury, ${ }^{30}$ to which the use of a measure such as the ISST may be a valuable addition.

Limitations require highlighting, despite the results of this study demonstrating high absolute and relative testretest reliability. The sample utilized in the study was a convenience sample from a specific male, academy age elite football population. Data therefore may not be extrapolated to other genders, age groups, sports or non-sporting populations. Further investigation is required to determine whether equivalent findings exist. Because bilateral limb testing was utilized in accordance with Ransom et al. ${ }^{14}$ the authors acknowledge that bilateral limb deficit phenomenon may exist, but measures to minimize the likelihood were highlighted in the methodological approach. ${ }^{31}$

\section{CONCLUSION}

The results of this study indicate that the ISST is a reliable method for assessing maximal isometric PF in the soleus musculature of male elite academy footballers. These results suggest that the ISST may be beneficial for performance practitioners for profiling soleus function of athletes.

Submitted: February 04, 2021 CST, Accepted: October 21, 2021 CST 


\section{REFERENCES}

1. Ekstrand J, Waldén M, Hägglund M. Hamstring injuries have increased by $4 \%$ annually in men's professional football, since 2001: a 13-year longitudinal analysis of the UEFA elite club injury study. Br J Sports Med. 2016;50(12):731-737. doi:10.11 36/bjsports-2015-095359

2. Barnes C, Archer DT, Hogg B, Bush M, Bradley P. The evolution of physical and technical performance parameters in the English Premier League. Int J Sports Med. 2014;35(13):1095-1100. doi:10.1055/s-0034-137 5695

3. Hägglund M, Waldén M, Bahr R, et al. Methods for epidemiological study of injuries to professional football players: developing the UEFA model. $\mathrm{Br} J$ Sports Med. 2005;39(6):340-346. doi:10.1136/bjsm.20 $\underline{05.018267}$

4. López-Valenciano A, Ruiz-Pérez I, Garcia-Gómez A, et al. Epidemiology of injuries in professional football: a systematic review and mete-analysis. $\mathrm{Br} \mathrm{J}$ Sports Med. 2019;0(0):1-9.

5. Opar DA, Piatkowski T, Williams MD, Shield AJ. A novel device using the Nordic hamstring exercise to assess eccentric knee flexor strength: A reliability and retrospective injury study. J Orthop Sports Phys Ther. 2013;43(9):636-640. doi:10.2519/jospt.2013.4837

6. Rhodes D, McNaughton L, Greig M. The temporal pattern of recovery in eccentric hamstring strength post-soccer specific fatigue. Res Sports Med. 2018;27(3):339-350. doi:10.1080/15438627.2018.1523 $\underline{168}$

7. Hägglund M, Waldén M, Ekstrand J. Risk factors for lower extremity muscle injury in professional soccer: The UEFA injury study. Am J Sports Med.

2013;41(2):327-335. doi:10.1177/0363546512470634

8. van Dyk N, Witvrouw E, Bahr R. Interseason variability in isokinetic strength and poor correlation with nordic hamstring eccentric strength in football players. Scand J Med Sci Sports. 2018;28(8):1878-1887.

9. Werner BC, Belkin NS, Kennelly S, et al. Acute gastrocnemius-soleus complex injuries in national football league athletes. Orthop J Sports Med. 2017;5(1):1-6. doi:10.1177/2325967116680344

10. Mokhtarzadeh H, Yeow CH, Goh JCH, Oetomo D, Malekipour F, Lee PVS. Contributions of the soleus and gastrocnemius muscles to the anterior cruciate ligament loading during single-leg landing. J Biomech. 2013;46(11):1913-1920. doi:10.1016/j.jbiomech.201 $\underline{3.04 .010}$
11. Elias JJ, Faust AF, Chu YH, Chao EY, Cosgarea AJ. The soleus muscle acts as an agonist for the anterior cruciate ligament: An in vitro experimental study. Am J Sports Med. 2003;31(2):241-246. doi:10.1177/036354 $\underline{65030310021401}$

12. Schrodter E, Bruggemann GP, Willwacher S. Is soleus muscle-tendon unit behaviour related to ground-force application during the sprint start? Int J Sports Phys Perf. 2017;12(4):448-454.

13. van Mechelen W, Hlobil H, Kemper HC. Incidence, severity, aetiology and prevention of sports injuries. A review of concepts. Sports Med. 1992;14(2):82-99. do i:10.2165/00007256-199214020-00002

14. Ransom M, Saunders S, Gallo T, et al. Reliability of a portable fixed frame dynamometry system used to test lower limb strength in elite Australian football league players. J Sci Med Sport. 2020;23(9):826-830. $\underline{\mathrm{d}}$ oi:10.1016/i.jsams.2020.03.006

15. Dos'Santos T, Thomas C, Jones PA, McMahon JJ, Comfort $\mathrm{P}$. The effect of hip joint angle on isometric mid-thigh pull kinetics. J Strength Cond Res. 2017;31(10):2748-2757. doi:10.1519/jsc.00000000000 02098

16. Dos'Santos T, Jones PA, Comfort P, Thomas C. Effect of different onset thresholds on isometric midthigh pull force-time variables. J Strength Cond Res. 2017;31(12):3467-3473. doi:10.1519/jsc.00000000000 $\underline{01765}$

17. Goodwill AM, Pearce AJ, Kidgell DJ. Corticomotor plasticity following unilateral strength training. Muscle \& Nerve. 2012;46(3):384-393.

18. Halperin I, Williams K, Martin D, et al. The effects of attentional focusing instructions on force production during the isometric midthigh pull. $J$ Strength Cond Res. 2016;30(4):919.

19. Comfort P, Dos'Santos T, Beckham G, et al. Standardization and methodological considerations for the isometric midthigh pull. Strength Cond J. 2019;41(2):57.

20. Beckham G, Mizuguchi S, Carter C, et al. Relationships of isometric mid-thigh pull variables to weightlifting performance. J Sports Med Phys Fitness. 2013;53(5):573-581. 
21. Beckham G, Sato K, Santana H, Mizuguchi S, Haff GG, Stone MH. Effect of body position on force production during the isometric midthigh pull. $J$ Strength Cond Res. 2018;32(1):48-56. doi:10.1519/js c. 0000000000001968

22. Bland MJ, Altman D. Statistical methods for assessing agreement between two methods of clinical measurement. The Lancet. 1986;327(8476):307-310. d oi:10.1016/s0140-6736(86)90837-8

23. McGuigan MR, Cormack SJ, Gill ND. Strength and power profiling of athletes: Selecting tests and how to use the information for program design. Strength Cond J. 2013;35(6):7-14. doi:10.1519/ssc.00000000000 $\underline{00011}$

24. De Witt J, English K, Crowell J, et al. Isometric mid-thigh pull reliability and relationship to deadlift. J Strength Con Res. 2018;32(2):528-533. doi:10.1519/js c.0000000000001605

25. Haff GG, Stone M, O’Bryant HS, et al. Force-time dependent characteristics of dynamic and isometric muscle actions. 1997;11(4):269-272.

26. Schache AG, Crossley KM, Macindoe IG, et al. Can a clinical test of hamstring strength identify football players at risk of hamstring strain? Knee Surg Sports Traumatol Arthrosc. 2011;19:38-41.
27. Buckthorpe M, Wright S, Bruce-Low S, et al. Recommendations for hamstring injury prevention in elite football: translating research into practice. $\mathrm{Br} \mathrm{J}$ Sports Med. 2019;53(3):449-456. doi:10.1136/bjsport $\underline{\mathrm{s}-2018-099616}$

28. Kawamori N, Rossi S, Justice B, et al. Peak force and rate of force development during isometric and dynamic mid-thigh clean pulls performed at various intensities. J Strength Cond Res. 2006;20(3):483-491. $\underline{\mathrm{d}}$ oi:10.1519/00124278-200608000-00005

29. Nedelec M, McCall A, Carling C, Legall F, Berthoin $S$, Dupont G. The influence of soccer playing actions on the recovery kinetics after a soccer match. $J$ Strength Cond Res. 2014;28(6):1517-1523. doi:10.151 9/jsc.0000000000000293

30. McCall A, Carling C, Davison M, et al. Injury risk factors, screening tests and preventative strategies: A systematic review of the evidence that underpins the perceptions and practices of 44 football (soccer) teams from various premier leagues. Br J Sports Med. 2015;49(9):583-589. doi:10.1136/bjsports-2014-09410 $\underline{4}$

31. Kuruganti U, Murphy T, Pardy T. Bilateral deficit phenomenon and the role of antagonist muscle activity during maximal isometric knee extensions in young, athletic men. Eur J App Physiol. 2011;111(7):1533-1539. doi:10.1007/s00421-010-175 $\underline{2-8}$ 